\title{
Study of the Recurrence Rate in the Treatment in Use Group of Acupuncture and Moxibustion and Non-in Use Group of a Breast Cancer Post-operatively
}

\author{
Takeo Mizushima*, Hideko Miyajima, Akiko Matsubara and Yosiko Kurihara \\ Mizushima clinic Sakushi Hara567-7 Nagano-pref, Japan \\ *Corresponding author: Takeo Mizushima Mizushima, Clinic Sakushi Hara567-7, Nagano-pref, Japan
}

Received: May 20, 2021; Accepted: May 31, 2021; Published: June 04, 2021

\begin{abstract}
There is a lot of recurrence of breast cancer post-operatively and prescription of an anticancer drug is necessary. But it's learned that treatment of acupuncture and moxibustion participates in autonomic nerve immunity. So the recurrence rate of 10 years later was considered while investigating a white-blood cell, the number of lymphocyte and monocytes, and TH1/TH2 and CA153, Treg cell every year and 10 years later to 51 cases which shared breast cancer patients of post-operatively with 2 groups. The first group is 30 cases used treatment of acupuncture and moxibustion and the second group is 21 cases of non-in use group, Treatment of acupuncture and moxibustion chose meridian treatment, Traditional Chinese Medicine treatment, Ono system treatment and Nagano system treatment from a pluses diagnosis according to each constitution. A result increased in a lymphocyte and TH1/TH2 ratio significantly 10 years later from the treatment front to non-in use group by a treatment group of acupuncture and moxibustion and T reg cell decreased 10 years later. The recurrence rate didn't recognize a significant difference stage 1 and Ruminull A and B by low value, but a treatment in use group of acupuncture and moxibustion made recurrence decrease predominantly to non-in use group by stage 2, 3, 4 and HER2 extrovert group.
\end{abstract}

Keywords: Breast cancer post-operatively, TH1/TH2 ratio recurrence rate, Acupuncture and moxibution treatment Treg cell

\section{Introduction}

In Japan a survival rate is $90.6 \%$ for breast cancer postoperative ten years on stage 1 . But stage 2 is $78.5 \%$ stage 3 is $33.0 \%$ stage 4 become $10.9 \%$. Of course, a new anticancer agent and an antihormone drugs are developed. But I did not know drugs for effective cancer immunity. Acupuncture and moxibution treatment have participates in autonomic nerve immunity. We provide postoperative breast cancer patients, the first group was operated standard chemotherapy with non-use treatment of acupuncture which are 21 cases, and the second group was operated with treatment of acupuncture and moxibution which are 30 cases and we investigate cancer immunity for WBC TH1/TH2 CA153 Treg cell every year and 10 yaers later. And two groups comparative investigate the rate of recurrence.

\section{Patient Target}

Informed consent was got 51 patients of post-operative of breast cancer. One group 21 cases were admitted standard chemotherapy and second group 30 cases was admitted standard chemotherapy with acupuncture and moxibution treatment.

\section{Approach}

51 cases we investigate WBC count and Lymphocyte Monocyte percentage, TH1/TH2 ratio, Treg cell count (normal value $8.0 \sim 34.0 \%$ ) and the presence of recurrence every year for 10 years later.

\section{Acupuncture Method}

Acupuncture and moxibution treatment have two effectiveness for human body [1,2]. One is sedative pain for sympathetic nerve and second is immunity effectiveness for parasympathetic nerve. We stimulate immunity effective that we stimulate disposal acupuncture needle $(\varphi 0.02 \mathrm{~mm}$ ) in depth 3-4 $\mathrm{mm}$ from the skin. And acupuncture point we select according pulse diagnosis traditional Chinese theory and Japanese nagano system, Ono system and meridian diagnosis system. For example, the nagano system suggest blood stasis stimulate tyuuhou (LV4) and syakutaku (L6), liver meridian emptiness stimulate eyou (B35) daityouyu (B25). Ono system suggest neck tenderness diagnosis tenntyuu (B10) is kidney meridian emptiness stimulate taikei (K3) and huyou (B59). Chinese traditional medicine suggest kidney meridian emptiness stimulate hukuryuu (K7) and keikyo (L6) [3-5]

\section{Examination}

For every year we examinate white blood cell count and lymph/ monocyte percent, $\mathrm{TH} 1 / \mathrm{TH} 2$ ratio, Treg cell count.

And breast cancer marker CA153 and the last existence of recurrence of local and distance for MRI and CT 10 years later.

\section{Statistics}

Significant difference between two groups for mann-whitney's method. 
Takeo Mizushima (2021) Study of the Recurrence Rate in the Treatment in Use Group of Acupuncture and Moxibustion and Non-in Use Group of a Breast Cancer Post-operatively

\section{Result}

Group 1 (30 cases) enforced chemotherapy with acupuncture and moxibution treatment

First diagnosis WBC: $3080 \pm 882 \mu / \mathrm{mL}$, lymphocyte/ monocyte: $20.2 \pm 4.6 / 6.8 \pm 2.4 \% \mathrm{TH} 1 / \mathrm{TH} 2: 20.4 \pm 12.8 / 4.9 \pm 3.8$ (ratio4.1) CA15-3:15.8 $\pm 6.8 \mu / \mathrm{mL}$, Treg: $58.0 \pm 14.8 \%$. 1 year later WBC: $3409 \pm 1084 \mu / \mathrm{mL}$ Ly/Mono:28.6 \pm 35.5/4.5 $\pm 43.8 \%$, TH1/ TH2:24.8 \pm 3.8/3.0 \pm 0.8 (ratio8.2), CA15-3: $12.4 \pm 3.8 \mathrm{u} / \mathrm{mL}$ Treg: $49.2 \pm 20.1 \% .5$ years later WBC: $3808 \pm 1209 \mu / \mathrm{mL}$, Ly/Mono:30.4 \pm $6.1 / 3.8 \pm 2.0^{*}, \mathrm{TH} 1 / \mathrm{TH} 2: 30.2 \pm 3.8 / 3.0 \pm 0.8$ (ratio7.9) ${ }^{* *} \mathrm{CA} 15-3: 10.8$ $\pm 2.9 \mathrm{u} / \mathrm{mL}$, Treg: $20.2 \pm 13.4 \%^{* * *} .10$ years later WBC: $3804 \pm 2004$ $\mu / \mathrm{mL}$ Ly/Mono:31.2 \pm 4.2/3.2 $\pm 1.6 \%, \mathrm{TH} 1 / \mathrm{TH} 2: 31.4 \pm 6.8 / 3.0 \pm 0.5$ (ratio10.4) $※$ CA15-3:10.8 $\pm 2.9 \mathrm{u} / \mathrm{mL}$, Treg : $8.6 \pm 5.5 \% ※ ※ \%$.

We saw significant difference 5 years later and 10 years later for the first diagnosis examination about lymphocyte/monocyte TH1/ $\mathrm{TH} 2$ ratio and Treg cell. And recurrence rate stage I cases (16 cases average age 63.0 y.o.) was $0 \%$ and stage II IV cases (14 cases average age 60.8 y.o.) saw 3 cases recurrence and its ratio was $21.4 \%$.

Second group was 21 cases enforced chemotherapy with nonacupuncture treatment.

First diagnosis: WBC: $2897 \pm 998 \mu / \mathrm{mL}$, Ly/Mono1 $8.9 \pm 3.9 \%$, TH1/TH2 : $20.6 \pm$ 8.8/5.2 \pm 3.0 (ratio 6.8) CA15-3:28.9 $\pm 4.7 \mathrm{u} /$ mL Treg: $55.2 \pm 10.8 \% .5$ years later : WBC:3208 $\pm 1090 \mu / \mathrm{mL}$ Ly/ Mono:20.8 \pm 6.0/6.1 $\pm 3.6 \%$, TH1/TH2 : $20.6 \pm 8.8 / 5.2 \pm 3.8$ (ratio3.9) CA15-3:28.9 $\pm 5.0 \mathrm{u} / \mathrm{mL}$, Treg: $52.4 \pm 21.4 \% .10$ years later : WBC:3200 $\pm 826 \mu / \mathrm{mL}$, Ly/Mono: $18.4 \pm 4.4 / 5.8 \pm 4.0 \% \mathrm{TH} 1 / \mathrm{TH} 2: 18.4 \pm 4.0 / 5.0 \pm$ 3.8 (ratio4.8) CA15-3:20.4 $\pm 3.8 \mathrm{u} / \mathrm{mL}$ Treg: $50.2 \pm 26.8 \%$

We saw TH1/TH2 Treg cell no difference between every examination and recurrence ratio was stage I group ( 9 cases ave. age 66.3 y.o.) $11.1 \%$. 1 case was seen bone metastasis. And stage II IV 12 cases (ave. age59.8 y.o.) was seen 8 cases recurrence (66.6\%) 10 years later (Figure 1).

Stage 1

cases ave.age recurrence rate

With Acupuncture and moxibution

$16 \quad 63.0 \quad 0$ case $(0 \%)$

With non-acupuncture

$9 \quad 66.3 \quad 1$ case $(11.1 \%)$

Stage II IV

cases ave.age recurrence rate

With acupuncture and moxibution

$14 \quad 60.8 \quad 3 \operatorname{cases}(21.4 \%)$

With non-acupuncture

$12 \quad 59.8 \quad 8 \operatorname{cases}(66.6 \%)$

We saw significant difference to a relapse rate in an acupuncture treatment group and non-acupuncture group for stage II IV ten years later. (Relative risk $\mathrm{p}<0.320$ ).

The rise in ratio of TH1/TH2 and the drop of the Treg cell were in particular remarkable. (Relative risk $\mathrm{p}<0.021$ ) and we saw Ruminal type A $\cdot$ B (HER2 negative) acupuncture treatment group are 18 cases seen recurrence 0 case $(0 \%)$ but non-acupuncture group (11 cases) was seen recurrence 2 cases (18.1\%) and HER2 positive group

Citation: acupuncture treatment (12 cases) was seen recurrence 3 cases (25\%) but non-acupuncture treatment (10 cases) was seen recurrence 7 cases $(70 \%)$ for 10 years later (relative risk $\mathrm{p}<0.435$ ) (Figure 2).
HER2 negative Ruminal A • B

With acupuncture and moxibution With non- acupuncture

HER2 positive

With acupuncture and moxibution With non- acupuncture cases ave.age recurrence rate

$$
\begin{array}{lll}
18 & 61.4 & 0 \text { case }(0 \%) \\
11 & 59.0 & 2 \operatorname{cases}(18.1 \%)
\end{array}
$$

cases ave.age recurrence rate$$
10 \quad 62.3 \quad 7 \text { cases }(70.0 \%)
$$

$$
12 \quad 60.8 \quad 3 \operatorname{cases}(25.0 \%)
$$

\section{Consideration}

Standard chemotherapy is done after operation of the breast cancer, but the relapse rate is not low at all. It is thought that it has a drop of the cancer immunity and participation of the Treg cell. TH1 cell conduct killer T cell with the help of cytokine INF $\gamma$, IL- 6 and TH2 cell disturb a function of cytokine [6-8]. In other words, we can induce cancer immunity will if the TH1/TH2 ratio is high. In addition, it is thought the Treg cell controls an effect of the chemotherapy [9-11]. We pay attention of the parasympathetic nerve of the acupuncture and moxibution treatment, I added acupuncture and moxibution treatment to the breast cancer postoperative cases. We thought the result acupuncture and moxibution treatment group watched the rise in $\mathrm{TH} 1 / \mathrm{TH} 2$ ratio and drop of the Treg and reduced a relapse rate in predominance.

\section{Conclusion}

It can let cancer immunity have the top by a rise of TH1/TH2 and the drop of the Treg cell to use acupuncture and moxibustion treatment together during breast cancer postoperative chemotherapy and can reduce a recurrence in its turn. You should use acupuncture and moxibustion treatment together during chemotherapy.

\section{References}

1. 水嶋丈雄著. 鍼炎治療への科学的アプローチ. 三和書籍. 2012: (Mizushima T.: Scientific approach of acupuncture and moxibution).

2. 首藤伝明著. 経絡治療のすすめ. 医道の日本社. 2015: 97-120. (Syudou D.: The advice for meridian treatment of acupuncture).

3. 陸寿康著．鍼炎手技学．東洋学術出版社．1992: 100-120. (Riku K.: Maneuver study of acupuncture).

4. 哀中会編. 臓腑経絡・ 三焦の弁証と処方. 医歯薬出版. 2000: 263-273. (Demonstration of Entrail and meridian of traditional chinese medicine).

5. 小野文恵著．鍼炎臨床入門．医道の日本社．1988：10-28．（Oon.B.： Medical practice of acupuncture and moxibution).

6. Sakaguchi S (2005) Nature Immunology 6(4): 345-352. [crossref]

7. De Oliveira, CE et al, (2017) Molecular Cancer Therapeutics, September.

8. Zwang NA, Leventhal JR (2017) Journal of the American Society of Nephrology 28(7). [crossref]

9. Ohmi Y (1996) Tumor-specific targeting of T helper type I (TH1) cells by anti-CD3 Xanti-c ErbB-2 bispecific antibody Cancer Immunol Immunother 48: 456-452. [crossref]

10. Nishimara T (1999) Distinct roll on antihen-specific T helper type I (Th1) and Th2 cells in tumor eradication in vivo. Exp Med 190.617-628. [crossref]

11. Kitamura H (1999) The natural killer Tcell ligand $\alpha$-galactosylceramide demonstrates its immnnopotentiating effect by inducing IL-12 production by dendritic cells and IL-12 receptor expression on NKT cell J Med 190.1121-1127. [crossref]

Mizushima T, Miyajima H, Matsubara A, Kurihara Y (2021) Study of the Recurrence Rate in the Treatment in Use Group of Acupuncture and Moxibustion and Non-in Use Group of a Breast Cancer Post-operatively. J Clin Res Med Volume 4(3): 1-2. 